\title{
miR-590-5p suppresses osteosarcoma cell proliferation and invasion via targeting KLF5
}

\author{
WEI CAI ${ }^{1}$, YONG XU $^{1}$, JIAN YIN $^{2}$, WENSHAN ZUO ${ }^{1}$ and ZHEN SU ${ }^{3}$ \\ ${ }^{1}$ Orthopedics Department, The Affiliated Huaian No. 1 People's Hospital of Nanjing Medical University, Huai'an, \\ Jiangsu 223300; ${ }^{2}$ Orthopedics Department, The Affiliated Jiangning Hospital, Nanjing Medical University, \\ Nanjing, Jiangsu 211100; ${ }^{3}$ Anesthesiology Department, The Affiliated Huaian No. 1 People's Hospital \\ of Nanjing Medical University, Huai'an, Jiangsu 223300, P.R. China
}

Received February 2, 2018; Accepted May 22, 2018

DOI: $10.3892 / \mathrm{mmr} .2018 .9173$

\begin{abstract}
Recently, microRNA (miR)-590-5p has been shown to inhibit tumorigenesis in colorectal and breast cancer; however, its function in osteosarcoma (OS) requires further investigation. In the present study miR-590-5p expression was poorly expressed in OS samples and cell lines when compared with that observed in normal cells. In addition, overexpression of miR-590-5p significantly reduced the proliferation, migration and invasion of SAOS2 and U2OS cells in vitro, as well as inhibiting tumor sizes in vivo. The results revealed that miR-590-5p directly targeted Kruppel-like factor 5 (KLF5) in SAOS2 and U2OS cells. Their expression was inversely correlated with OS tissues. Finally, it was demonstrated that overexpression of KLF5 rescued the inhibitory effects of miR-590-5p on cell proliferation, migration and invasion. Overall, the results of the present study suggested that the miR-590-5p/KLF5 axis may regulate OS progression and thus, may be a novel therapeutic target for the treatment of patients with OS.
\end{abstract}

\section{Introduction}

Osteosarcoma (OS) is a highly aggressive and common primary bone tumor that often occurs among young individuals worldwide $(1,2)$. Although considerable effort has been made to develop effective methods for OS therapy, the clinical outcome of patients with OS has not improved. In particular, the 5-year survival rate of patients with metastatic or advanced OS is lower than $30 \%(3,4)$. Every

Correspondence to: Professor Zhen Su, Anesthesiology Department, The Affiliated Huaian No. 1 People's Hospital of Nanjing Medical University, 6 Beijing West Road, Huai'an, Jiangsu 223300, P.R. China

E-mail: suzhen_nju@163.com

Key words: microRNA-590-5p, Kruppel-like factor 5, proliferation, migration, osteosarcoma year, OS accounts for a large percentage of cancer-related deaths worldwide (5). Thus, understanding the underlying mechanism and identifying new therapeutic targets for OS treatment are necessary.

MicroRNAs (miRNAs/miRs) are a class of short RNAs that have a length of 21-25 nucleotides and no protein-coding ability (6). A study on their mechanism demonstrated that miRNAs can regulate target gene expression by associating with the 3'-untranslated region of miRNAs and directing its degradation (7). In recent decades, increasing evidence indicated that miRNAs are generally involved in diverse biological processes, such as cell proliferation, differentiation, and death (8-10). Dysregulation of miRNAs is proven to be closely related to human diseases, especially tumor development and progression (11). For instance, miR-589-3p regulates cell migration and invasion in glioblastoma (12), and miR-29c-3p participates in the regulation of colorectal cancer (CC) growth (13). In addition, miRNA-139 can inhibit the progression of papillary thyroid carcinoma (14). An increasing number of reports have shown that miRNAs are good biomarkers for cancer diagnosis, prognosis, and treatment $(15,16)$. Therefore, fully understanding the underlying molecular mechanism by which miRNAs regulate tumorigenesis will benefit cancer intervention.

miR-590-5p has been reported to inhibit tumor growth in CC by targeting YAP1 (17). Another study showed that miR-590-5p suppresses stemness and metastasis in breast cancer by targeting SOX2 (18). However, the function and mechanism of miR-590-5p on OS remain to be elucidated.

In our study, we showed that miR-590-5p was lowly expressed in OS tissues and cell lines compared with that in normal tissues or cells. In addition, overexpression of miR-590-5p in SAOS2 and U2OS cells inhibited cell proliferation, migration, and invasion. We found that miR-590-5p could suppress OS cell entry into the cell cycle. Mechanistically, we observed that miR-590-5p directly targeted KLF5, which is a transcription factor belonging to the Kruppel-like factor subfamily of zinc finger proteins, in SAOS2 and U2OS cells. Finally, restoration of KLF5 in miR-590-5p-overexpressing SAOS2 and U2OS cells rescued proliferation, migration, and invasion. In summary, our finding indicated that miR-590-5p suppressed OS progression by targeting KLF5. 


\section{Materials and methods}

Clinical specimens. For this study, we collected 51 OS samples and 19 adjacent normal tissues from Huai'an First People's Hospital, Nanjing Medical University (Huai'an, China). None of the patients had received any adjuvant chemotherapy or radiotherapy before surgery. Matched adjacent noncancerous tissues were collected $>5 \mathrm{~cm}$ away from the tumors and were verified by two independent pathologists at the same time. We obtained written informed consents that approved the usage of these sample tissues in this study from all patients. All of the experiments were approved by Huai'an First People's Hospital, Nanjing Medical University. The study protocol was approved by Huai'an First People's Hospital, Nanjing Medical University.

Cell culture and transfection. Human osteoblast cell line (hFOB1.19) and OS cell lines including U2OS, MG63 and SAOS2 were purchased from American Type Culture Collection. Cells were cultured in the DMEM medium supplemented with $10 \%$ fetal bovine serum (HyClone; GE Healthcare Life Sciences, Logan, UT, USA) and anti-biotics in a humidified containing of $5 \% \mathrm{CO}_{2}$ incubator at $37^{\circ} \mathrm{C}$.

miR-590-5p mimics, inhibitors and corresponding negative controls were purchased from Genecopoeia (Guangzhou, China) and transduced into cells with Lipofectamine 2000 (Invitrogen; Thermo Fisher Scientific, Inc., Waltham, MA, USA) according to the manufactures' instruction. For KLF5 overexpression, the coding sequence of KLF5 was cloned into pCDNA3-vector. And then pCDNA3-KLF5 was transfected into OS cells using Lipofectamine 2000 (Invitrogen; Thermo Fisher Scientific, Inc.) according to the manufactures' instruction.

Cell proliferation assay. $1 \times 10^{4}$ cells/well were seeded in 96-well plates and cultured. Cell proliferation was measured by a CCK8 cell proliferation assay every other day. $10 \mu \mathrm{l}$ CCK8 solution was added and incubated for $2 \mathrm{~h}$ at $37^{\circ} \mathrm{C}$. Then the absorbance was analyzed at $450 \mathrm{~nm}$ using SUNRISE Microplate Reader (Tecan Group, Ltd., Mannedorf, Switzerland).

Migration and invasion assay. $2 \times 10^{3}$ cells/well were seeded into the upper chamber of 24-well chambers and cultured in serum-free medium. The lower chamber was added medium with $10 \%$ FBS. 48 h later, migrating cells were fixed and stained with $0.5 \%$ crystal violet. Then three randomly fields were counted with a microscope. For invasion assay, the seeded cells were pre-coated with $500 \mathrm{ng} / \mathrm{ml}$ Matrigel (BD Biosciences, Franklin Lakes, NJ, USA). Other steps were the same as migration assay.

Reverse transcription-quantitative polymerase chain reaction (RT-qPCR). Total RNAs were isolated using TRIzol reagent from OS samples according to the manufacturer's protocol. cDNA were synthesized and used for analysis of mRNA transcripts using ABI 7300 qPCR system. RT-qPCR was performed with the SYBR green Premix Ex Taq II (Takara Bio, Inc., Otsu, Japan) on an Applied Biosystems Step One Plus Real-Time PCR System (Applied Biosystems; Thermo Fisher Scientific, Inc.). The thermocycling conditions were as follows: An initial denaturation step at $95^{\circ} \mathrm{C}$ for $5 \mathrm{~min}$, followed by
40 cycles of $95^{\circ} \mathrm{C}$ for $10 \mathrm{sec}, 60^{\circ} \mathrm{C}$ for $20 \mathrm{sec}$ and $72^{\circ} \mathrm{C}$ for $10 \mathrm{sec}$. Relative expressions were calculated and normalized to endogenous $A C T B$. Relative gene expression level was calculated using the $2^{-\Delta \Delta \mathrm{Cq}}(19)$

Colony formation assay. SAOS2 and U2OS cells were trypsinized into a single-cell suspension, and plated in each well of the 6-well plate and cultured for 14 days. The colonies were then washed twice with phosphate-buffered saline (PBS), fixed with $4 \%$ paraformaldehyde, and stained with $0.1 \%$ crystal violet. The number of colonies formed was imaged and counted under a light microscope (Olympus Corporation, Tokyo, Japan).

Cell cycle analysis. The cell cycle stage was determined using the Cell Cycle Analysis kit (Beyotime Institute of Biotechnology, Shanghai, China) following the manufacturer's instructions. Transfected cells were seeded into 6-well plates for $48 \mathrm{~h}$. Cells were then harvested and washed three times with cold PBS and fixed in $70 \%$ ethanol in PBS at $-20^{\circ} \mathrm{C}$ for $2 \mathrm{~h}$, followed by washing with PBS and staining the fixed cells with $50 \mu \mathrm{g} / \mathrm{ml}$ propidium iodide for $30 \mathrm{~min}$ in the dark at $37^{\circ} \mathrm{C}$. Analyses were performed on a FACS Calibur flow cytometer (BD Biosciences) using the ModFit software (BD Biosciences).

Western blotting. Protein from the tissues or cultured cells was extracted on ice in radioimmunoprecipitation assay lysis buffer containing protease inhibitor (Beyotime Institute of Biotechnology) for $30 \mathrm{~min}$. Equal amounts of protein were separated by $10 \%$ sodium dodecyl sulfate-polyacrylamide gel electrophoresis and transferred to nitrocellulose membranes (GE Healthcare, Chicago, IL, USA). Following blocking in 5\% non-fat milk in TBS, the membranes were incubated with the following antibodies: Anti-CYCLIN D1 (no. 2922; Cell Signaling Technology, Inc., Danvers, MA, USA), anti-CYCLIN-E1 (no. 20808; Cell Signaling Technology, Inc.), anti-p21 (no. 2947; Cell Signaling Technology, Inc.), anti-KLF5 (no. 51586; Cell Signaling Technology, Inc.) and anti-GAPDH (no. 5174; Cell Signaling Technology, Inc.) overnight at $4^{\circ} \mathrm{C}$. The membranes were then washed with TBST, and incubated with a goat anti-mouse (or rabbit $\mathrm{IgG}$ ) conjugated with horseradish peroxidase substrate (Santa Cruz Biotechnology, Inc., Dallas, TX, USA) for $1 \mathrm{~h}$ at room temperature. GAPDH was used as an internal control. The protein bands were detected with a chemiluminescent detection system (Beyotime Institute of Biotechnology).

Tumor xenograft. Six-week old BALB/c nude mice were obtained from HFK Biosciences. Each mouse was subcutaneously injected with $2 \times 10^{6}$ MG63 cells on the left flank region. The tumor volumes were measured at indicative time points. After 30 days, the weights of tumors were analyzed. Animal experiments were performed in accordance with relevant guidelines and regulations of the Institutional Animal Care and Use Committees at Huai'an First People's Hospital, Nanjing Medical University.

Luciferase reporter assay. Wild type (WT) or mutant (MUT) 3'-UTR of KLF5 was amplified and cloned into a pmiR-RB-REPORTTM Luciferase vector. Then, the WT or 
Table I. Associations between clinicopathological features and the expression of miR-590-5p in osteosarcoma tissues.

\begin{tabular}{|c|c|c|c|}
\hline Feature & $\begin{array}{c}\text { miR-590-5p } \\
\text { low expression }(n)\end{array}$ & $\begin{array}{c}\text { miR-590-5p } \\
\text { high expression (n) }\end{array}$ & P-value \\
\hline All cases & 27 & 24 & \\
\hline Age (years) & & & 1.000 \\
\hline$<20$ & 17 & 16 & \\
\hline$\geq 20$ & 10 & 8 & \\
\hline $\begin{array}{l}\text { Tumor size } \\
(\mathrm{cm})\end{array}$ & & & 0.025 \\
\hline$<5$ & 10 & 17 & \\
\hline$\geq 5$ & 17 & 7 & \\
\hline Metastases & & & 0.026 \\
\hline No & 8 & 15 & \\
\hline Yes & 19 & 9 & \\
\hline $\begin{array}{l}\text { Clinical } \\
\text { stage }\end{array}$ & & & 0.041 \\
\hline $\mathrm{I} / \mathrm{II}$ & 13 & 19 & \\
\hline III & 14 & 5 & \\
\hline
\end{tabular}

The median expression level was used as the cutoff. Results were considered statistically significant at $\mathrm{P}<0.05$. miR, microRNA.

MUT 3'-UTR of KLF5 as well as miR-590-5p mimic or negative control was co-transfected into cells by Lipofectamine 2000 (Invitrogen). $48 \mathrm{~h}$ after co-transfection, reporter activity was detected using a Dual-Luciferase ${ }^{\circledR}$ Reporter Assay kit (Promega Corporation, Madison, WI, USA).

Statistical analysis. All statistical analyses were performed using SPSS 20.0 (IBM Corp., Armonk, NY, USA) and GraphPad Prism 5.0 (GraphPad Software, Inc., La Jolla, CA, USA). Student's t-test and one-way analysis of variance followed by Tukey's post hoc test were used to analyze 2 or multiple groups, respectively, for statistical significance. Pearson correlation coefficient analysis was used to determine the correlations. $\mathrm{P}<0.05$ was considered to indicated a statistically significant difference.

\section{Results}

miR-590-5p was downregulated in OS. To determine the role of miR-590-5p in OS, we first checked its expression patterns in OS tissues. We performed RT-qPCR and found that miR-590-5p was downregulated in OS tissues $(n=51)$ compared with that in adjacent normal tissues $(n=19$; Fig. 1A and B). Moreover, miR-590-5p was expressed higher in non-metastatic OS tissues than in metastatic tissues (Fig. 1C). We then analyzed the clinicopathological characteristics of the 51 OS samples. As shown in Table I, the expression of miR-590-5p was inversely correlated with tumor size, metastasis, and clinical stages. Finally, we checked the expression of miR-590-5p in OS cell lines by RT-qPCR and found that miR-590-5p was also downregulated in SAOS2, U2OS, and MG63 cells compared with that in hFOB1.19 cells (Fig. 1D).
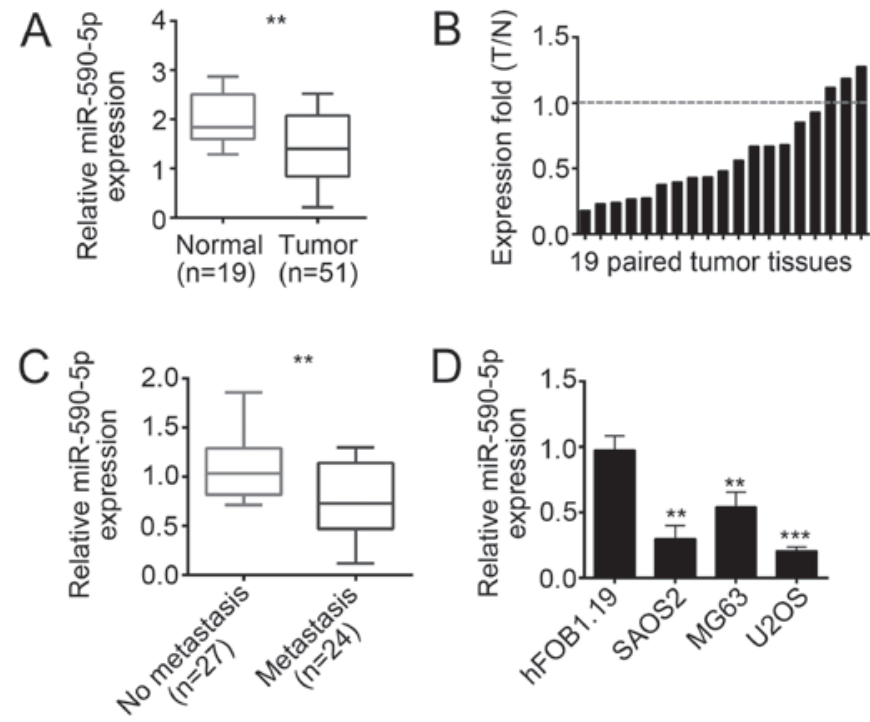

Figure 1. miR-590-5p is downregulated in OS. (A) RT-qPCR was used to determine the expression of miR-590-5p in OS tissues $(n=51)$ and adjacent normal tissues $(n=19)$. (B) Expression of miR-590-5p in 19 paired OS tissues and normal tissues was examined by RT-qPCR. (C) Expression of miR-590-5p was evaluated by RT-qPCR in OS tissues with and without metastasis. (D) Expression of miR-590-5p determined by RT-qPCR in OS cell lines. The results represent three independent experiments and are expressed as mean \pm standard deviation. ${ }^{* *} \mathrm{P}<0.01$ and ${ }^{* * *} \mathrm{P}<0.001$, vs. hFOB1.19/as indicated. OS, osteosarcoma; miR, microRNA; RT-qPCR, reverse transcription-quantitative polymerase chain reaction; $\mathrm{T}$, tumor; $\mathrm{N}$, normal tissue.

miR-590-5p overexpression inhibited SAOS2 and U2OS cellular proliferation, migration, and invasion. To analyze the role of miR-590-5p in OS cells, we overexpressed miR-590-5p in SAOS2 and U2OS cells by transducing miR-590-5p mimics (Fig. 2A). We conducted CCK8 and colony formation assays, and the results indicated that miR-590-5p overexpression significantly suppressed the proliferation of SAOS2 and U2OS cells (Fig. 2B and C). Furthermore, transwell assay revealed that miR-590-5p overexpression significantly inhibited the abilities of migration and invasion in SAOS2 and U2OS cells (Fig. 2D and E). To further verify the physiological function of miR-590-5p, we performed xenograft experiments. We found that miR-590-5p overexpression markedly suppressed tumor growth in vivo (Fig. 2F). At the end time point of the experiment, we measured the tumor weights and found that miR-590-5p dramatically reduced tumor sizes (Fig. 2G).

To further confirm the effect of miR-590-5p on OS cells, we suppressed miR-590-5p in U2OS and SAOS2 cells by transfection with miR-590-5p inhibitors (Fig. 2H). Then we performed CCK8 and transwell assays. Consistently, inhibition of miR-590-5p significantly promoted the proliferation, migration and invasion of SAOS2 and U2OS cells (Fig. 2I-K).

miR-590-5p overexpression arrested OS cells in G0 phase. To determine the mechanism of miR-590-5p-mediated inhibition of proliferation, we analyzed the effect of miR-590-5p on the cell cycle. miR-590-5p overexpression significantly arrested SAOS2 and U2OS cells in G0 phase (Fig. 3A and B). Consistently, the protein levels of CYCLIN D1 and CYCLIN E1 were downregulated after miR-590-5p overexpression (Fig. 3C). However, the protein level of p21 was upregulated 
A

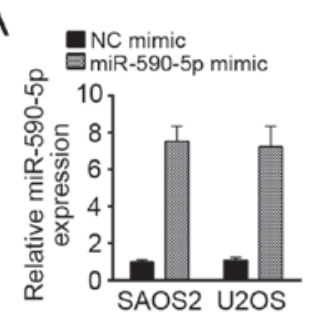

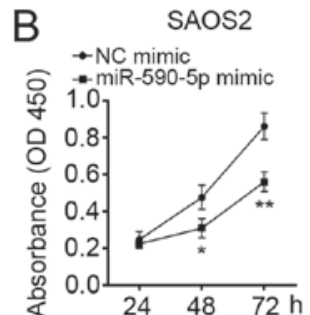
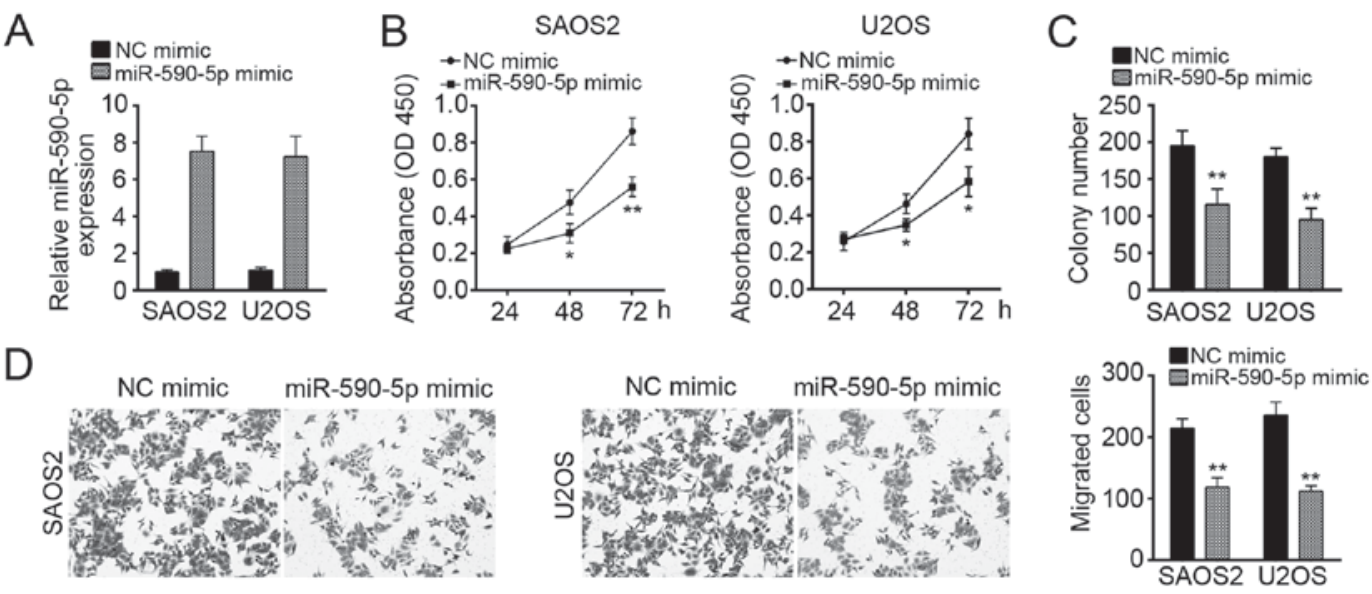

$\mathrm{E}$
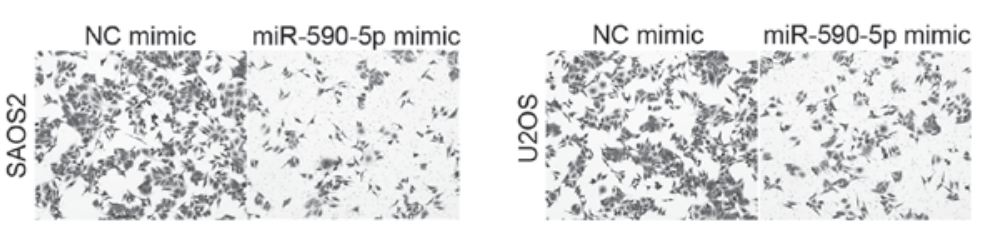

$\mathrm{F}$

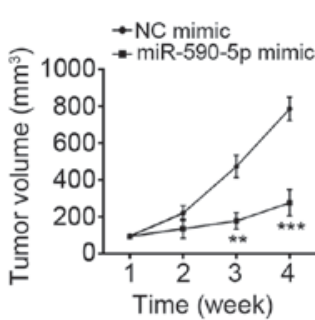

G
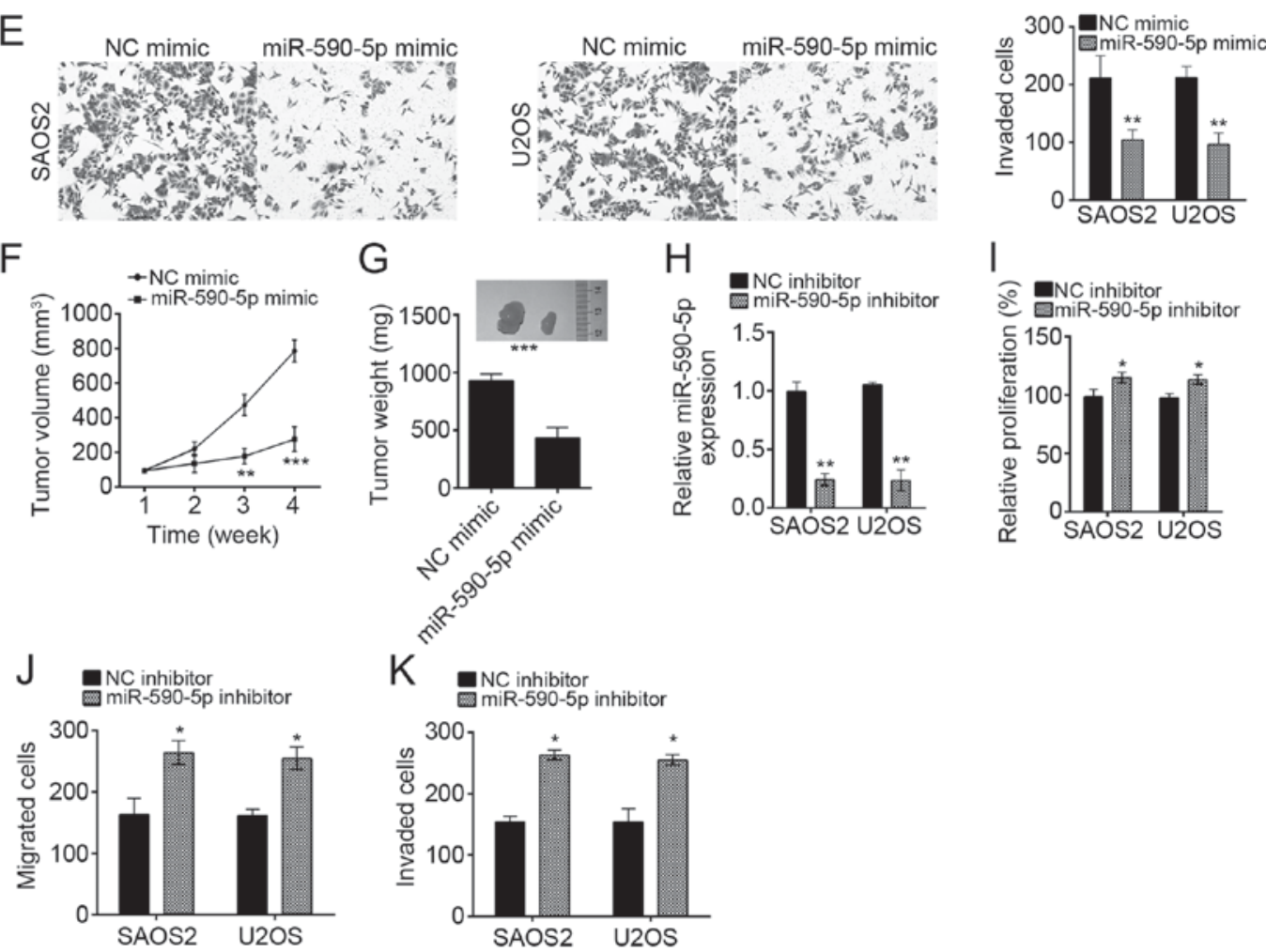

Figure 2. miR-590-5p overexpression inhibits SAOS2 and U2OS cellular proliferation, migration and invasion. (A) Overexpression of miR-590-5p was achieved by transducing miR-590-5p mimics in SAOS2 and U2OS cells. (B) CCK8 and (C) colony formation assays indicated that miR-590-5p overexpression inhibited the proliferation of SAOS2 and U2OS cells. Overexpression of miR-590-5p inhibited cell (D) migration and (E) invasion as demonstrated by transwell assay (magnification, x100). (F) Overexpression of miR-590-5p decreased tumor volumes. (G) miR-590-5p overexpression reduced tumor sizes. (H) Relative expression of miR-590-5p in SAOS2 and U2OS cells transfected with miR-590-5p inhibitor or control. (I) CCK8 assays were used to detect cell proliferation. Transwell assays were utilized to measure cell $(\mathrm{J})$ migration and $(\mathrm{K})$ invasion. The results represent three independent experiments and are expressed as the mean \pm standard deviation. ${ }^{*} \mathrm{P}<0.05,{ }^{* *} \mathrm{P}<0.01$ and ${ }^{* * *} \mathrm{P}<0.001$, vs. NC. CCK8, Cell Counting Kit-8; NC, negative control; miR, microRNA.

by miR-590-5p overexpression (Fig. 3C). Overall, miR-590-5p inhibited cell proliferation by reducing the cell cycle.

KLF5 was a target of miR-590-5p in OS. To explore the downstream target of miR-590-5p, we performed bioinformatics analysis (http://www.targetscan.org/vert_71/). We found that KLF5 was a potential target of miR-590-5p because of a potential binding site of miR-590-5p in the 3'-UTR of KLF5 mRNA (Fig. 4A). To confirm this prediction, we performed luciferase activity reporter assay. We observed that miR-590-5p overexpression significantly suppressed luciferase intensity in SAOS2 and U2OS cells transduced with WT 3'-UTR of KLF5 mRNA (Fig. 4B). When the binding site was mutated,
miR-590-5p overexpression had no effect on luciferase intensity (Fig. 4B), thereby suggesting that KLF5 was a direct target of miR-590-5p. Furthermore, overexpression of miR-590-5p remarkably decreased the mRNA and protein levels of KLF5 in SAOS2 and U2OS cells (Fig. 4C and D). Thus, the expression of KLF5 and miR-590-5p in OS tissues was inversely correlated (Fig. 4E).

Restoration of KLF5 in miR-590-5p-overexpressing SAOS2 and U2OS cells rescued cell proliferation, migration, and invasion. The role of KLF5 in OS has not been previously investigated. To define the role of KLF5 and determine whether miR-590-5p regulates OS by targeting KLF5, we overexpressed 
A

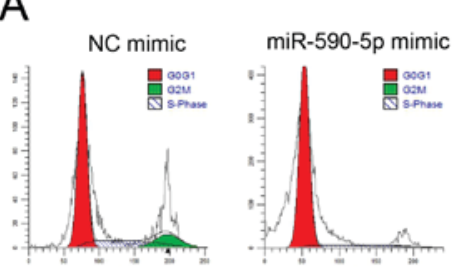

B
SAOS2

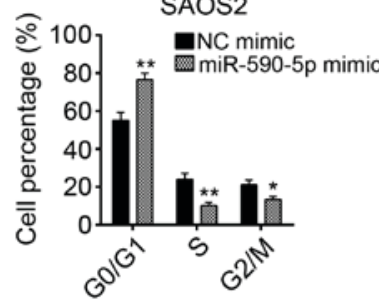

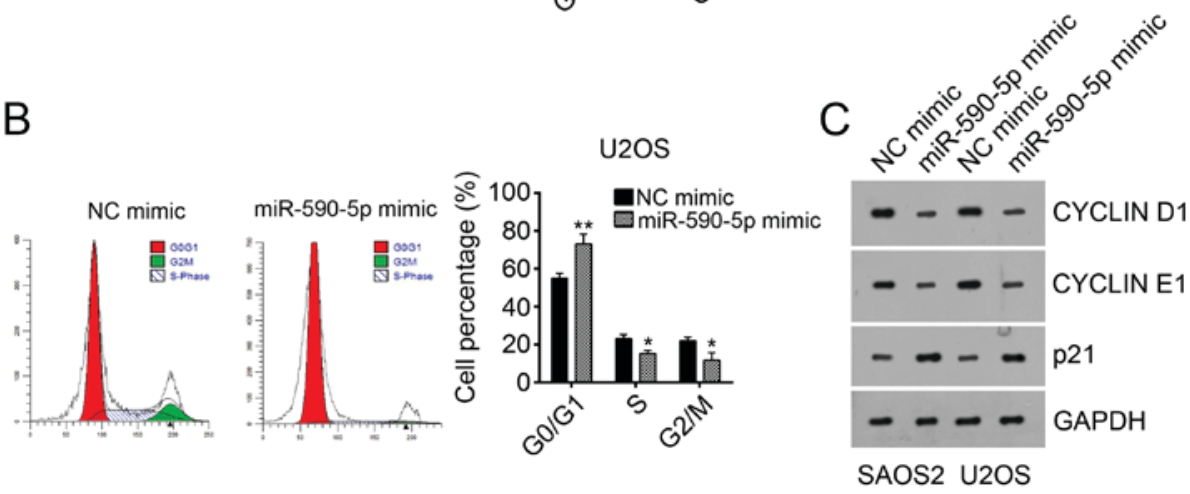

Figure 3. miR-590-5p overexpression arrests OS cells in the $\mathrm{G}_{0}$ phase. (A and B) Overexpression of miR-590-5p significantly increased the cells in the $\mathrm{G}_{0}$ phase, and reduced the cells in the $\mathrm{S}$ and $\mathrm{G}_{2} / \mathrm{M}$ phases. (C) Overexpression of miR-590-5p markedly inhibited the protein levels of Cyclin D1 and Cyclin E1, and promoted the levels of $\mathrm{p} 21$. The results represent three independent experiments and are expressed as the mean \pm standard deviation. ${ }^{*} \mathrm{P}<0.05$ and ${ }^{* * *} \mathrm{P}<0.01$ vs. NC mimic. NC, negative control; miR, microRNA; OS, osteosarcoma.

\section{A}

KLF5 WT: $5^{\prime}$-UACAAUAAGCUA-3' miR-590-5p: $3^{\prime}$-UACUUAUUCGAG-5' KLF5 MUT: $5^{\prime}$-UACAUAUUCGAA-3'

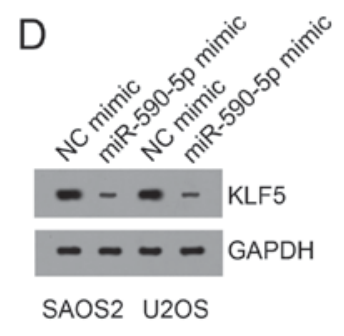

E
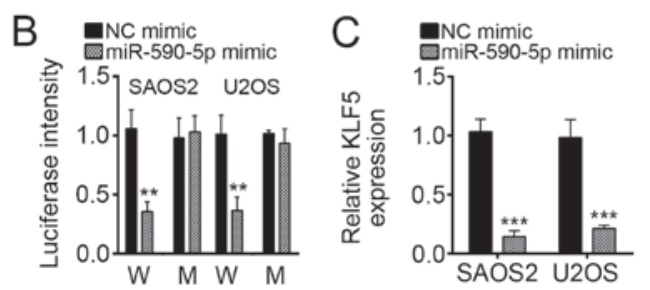

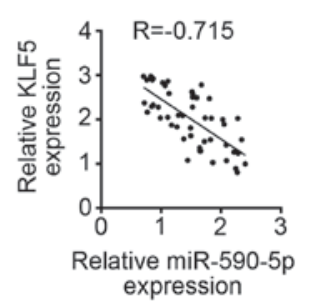

Figure 4. KLF5 is a target of miR-590-5p in OS. (A) Illustration of the potential binding site between miR-590-5p and the 3'-UTR of KLF5 mRNA. (B) miR-590-5p mimic inhibited luciferase intensity in SAOS2 and U2OS cells, whereas the mutation of the binding site in the 3'-UTR of KLF5 mRNA abrogated the effect of the miR-590-5p mimic. Overexpression of miR-590-5p significantly inhibited the (C) mRNA and (D) protein levels of KLF5 in SAOS2 and U2OS cells. (E) An inverse association between the expression of KLF5 and miR-590-5p in OS tissues was observed. The results represent three independent experiments and are expressed as the mean \pm standard deviation. ${ }^{* * *} \mathrm{P}<0.01$ and ${ }^{* * * *} \mathrm{P}<0.001$ vs. NC mimic. OS, osteosarcoma; NC, negative control; miR, microRNA; KLF5, Kruppel-like factor 5; W/WT, wild-type; M/MUT, mutant; UTR, untranslated region.
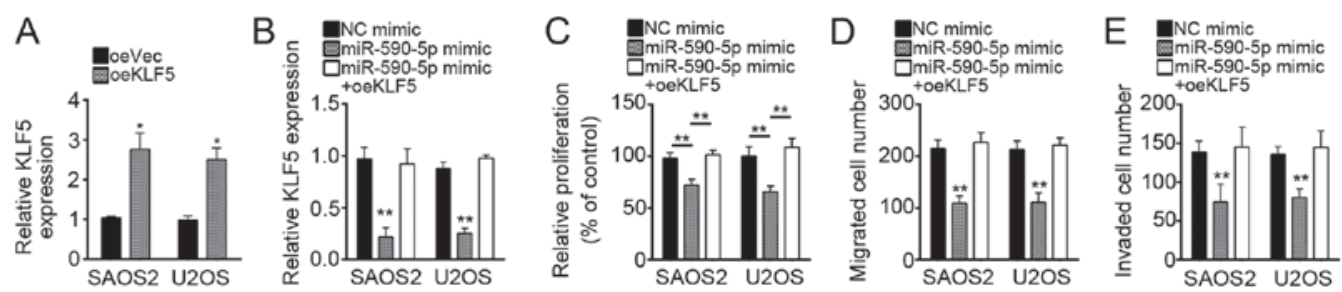

Figure 5. Restoration of KLF5 in miR-590-5p-overexpressing SAOS2 and U2OS cells rescues cell proliferation, migration and invasion. (A) RT-qPCR analysis for KLF5 mRNA levels in SAOS2 and U2OS cells transfected with pCNDA3-KLF5 or empty vector. (B) RT-qPCR analysis revealed that KLF5 expression was restored in miR-590-5p-overexpressing SAOS2 and U2OS cells. (C) Cell Counting Kit-8 results indicated that restoration of KLF5 rescued the proliferation ability of SAOS2 and U2OS cells. (D and E) Transwell assays demonstrated that restoration of KLF5 rescued the migration and invasion ability of SAOS2 and U2OS cells. The results represent three independent experiments and are expressed as the mean \pm standard deviation. ${ }^{*} \mathrm{P}<0.05$ and ${ }^{* * *} \mathrm{P}<0.01$ vs. NC mimic/as indicated. KLF5, Kruppel-like factor 5; miR, microRNA; RT-qPCR, reverse transcription-quantitative polymerase chain reaction; oe, overexpression; Vec, empty vector. 
KLF5 in miR-590-5p-overexpressing SAOS2 and U2OS cells to restore the level of KLF5 (Fig. 5A and B). We then conducted CCK8 and transwell assays. We found that restoration of KLF5 expression reversed the miR-590-5p-mediated inhibitory effect on cell proliferation, migration, and invasion (Fig. 5C-E). Overall, our results indicated that miR-590-5p inhibited OS cell proliferation, migration, and invasion by targeting KLF5.

\section{Discussion}

OS is the most malignant bone cancer worldwide. The clinical outcome of OS is extremely poor because of tumor metastasis and recurrence (20). Therefore, research on the molecular mechanism involved in OS development and progression, as well as screening of novel biomarkers and therapeutic targets, is necessary. Here, we found that miR-590-5p was downregulated in OS tissues and cell lines. We also identified KLF5 as a target gene of miR-590-5p in OS. miR-590-5p could suppress OS cell proliferation, migration, and invasion by targeting KLF5.

Numerous reports have indicated that dysregulation of miRNAs is closely associated with human cancers by targeting specific genes (21). miRNAs can regulate cell proliferation, apoptosis, and metastasis in various cancers $(22,23)$. For example, we previously reported that miR-367 can enhance the proliferation and metastasis of OS cells by targeting DAB2IP (24). Cui and Shi (25) reported that miR-187 suppresses OS growth and metastasis. Zhang and colleagues showed that miR-375 suppresses oral squamous cell carcinoma growth by targeting IGF-1R (26). Previous reports demonstrated that miR-590-5p can inhibit tumor growth in $\mathrm{CC}$ and breast cancer $(17,18)$. On the contrary, another study indicated that miR-590-5p promotes CC progression (27). Thus, the role of miR-590-5p in cancers must be illustrated further. Here, we revealed that miR-590-5p was lowly expressed in OS tissues and cell lines, suggesting that miR-590-5p may have an inhibitory effect on OS cells. The results of CCK8 and transwell assays proved that miR-590-5p overexpression significantly inhibited cell proliferation, migration, and invasion.

Kruppel-like factor 5 (KLF5) is a transcription factor that belongs to the Kruppel-like factor subfamily of zinc finger proteins. Previous research showed that KLF5 is involved in the regulation of cell proliferation and migration in various cancers (28). For instance, knockdown of KLF5 suppresses gastric cancer progression (29). Another study demonstrated that KLF5 promotes the proliferation, migration, and invasion of cervical cancer cells (28). KLF5 also promotes the growth, migration, and invasion of triple-negative breast cancer cells (30). Liu et al (31) revealed that KLF5 increases cell proliferation and invasion in human laryngeal squamous cell carcinoma. However, the function of KLF5 in OS remains largely unknown. Our study confirmed that KLF5 was a target gene of miR-590-5p in OS cells. Furthermore, CCK8 and transwell assays were conducted to reveal that KLF5 could rescue the abilities of proliferation, migration, and invasion in miR-590-5p-overexpressing SAOS2 and U2OS cells. The downstream signaling regulated by KLF5 remains unclear.
Tumor metastasis to other sites, such as in the lungs, is the main cause for OS-induced deaths (32). In our study, miR-590-5p was lowly expressed in metastatic OS tissues compared with that in metastatic tissues. Thus, miR-590-5p might be an ideal biomarker for OS clinical outcomes and a potential therapeutic target for OS treatment.

Collectively, our data demonstrated that miR-590-5p/KLF5 axis was a novel signal that regulated OS development and progression.

\section{Acknowledgements}

Not applicable.

\section{Funding}

No funding was received.

\section{Availability of data and materials}

All data generated or analyzed during this study are included in this published article.

\section{Authors' contributions}

ZS and WC conceived and designed the present study, analyzed and interpreted the results, and wrote the manuscript. YX, JY and WZ performed the experiments. All authors read and approved the final manuscript.

\section{Ethics approval and consent to participate}

For the use of human samples, the protocol for the present study was approved by the Institutional Ethics Committee of Huai'an First People's Hospital, Nanjing Medical University (Jiangsu, China) and all enrolled patients signed a written informed consent document. In addition, all procedures involving animals conformed to the national guidelines of, and were approved by, the Animal Care Ethics Committee of Nanjing Medical University.

\section{Consent for publication}

All patients recruited to the present study provided written informed consent for the publication of their data.

\section{Competing interests}

The authors declare that they have no competing interests.

\section{References}

1. Kansara M, Teng MW, Smyth MJ and Thomas DM: Translational biology of osteosarcoma. Nat Rev Cancer 14: 722-735, 2014.

2. Geller DS and Gorlick R: Osteosarcoma: A review of diagnosis, management, and treatment strategies. Clin Adv Hematol Oncol 8: 705-718, 2010

3. Xiong Y, Wu S, Du Q, Wang A and Wang Z: Integrated analysis of gene expression and genomic aberration data in osteosarcoma (OS). Cancer Gene Ther 22: 524-529, 2015.

4. Yuan W, Wang D, Liu Y, Tian D, Wang Y,Zhang R, Yin L and Deng Z: miR-494 inhibits cell proliferation and metastasis via targeting of CDK6 in osteosarcoma. Mol Med Rep 16: 8627-8634, 2017. 
5. Yang Z, Li X, Yang Y, He Z, Qu X and Zhang Y: Long noncoding RNAs in the progression, metastasis, and prognosis of osteosarcoma. Cell Death Dis 7: e2389, 2016.

6. Bartel DP: MicroRNAs: Genomics, biogenesis, mechanism, and function. Cell 116: 281-297, 2004.

7. Ivey KN and Srivastava D: MicroRNAs as regulators of differentiation and cell fate decisions. Cell Stem Cell 7: 36-41, 2010.

8. Gao X, Li S, Li W, Wang G, Zhao W, Han J, Diao C, Wang X and Zhang M: MicroRNA-539 suppresses tumor cell growth by targeting the WNT8B gene in non-small cell lung cancer. J Cell Biochem: Dec 21, 2017 (Epub ahead of print).

9. Shentu TP, Huang TS, Cernelc-Kohan M, Chan J, Wong SS, Espinoza CR, Tan C, Gramaglia I, van der Heyde H, Chien S and Hagood JS: Thy-1 dependent uptake of mesenchymal stem cell-derived extracellular vesicles blocks myofibroblastic differentiation. Sci Rep 7: 18052, 2017.

10. Huang G, Pan J, YeZ, Fang B, Cheng W and Cao Z: Overexpression of miR-216b sensitizes NSCLC cells to cisplatin-induced apoptosis by targeting c-Jun. Oncotarget 8: 104206-104215, 2017.

11. Rupaimoole R and Slack FJ: MicroRNA therapeutics: Towards a new era for the management of cancer and other diseases. Nat Rev Drug Discov 16: 203-222, 2017.

12. Cesarini V, Silvestris DA, Tassinari V, Tomaselli S, Alon S, Eisenberg E, Locatelli F and Gallo A: ADAR2/miR-589-3p axis controls glioblastoma cell migration/invasion. Nucleic Acids Res 46: 2045-2059, 2018.

13. Zhang S, Jin J, Tian X and Wu L: hsa-miR-29c-3p regulates biological function of colorectal cancer by targeting SPARC. Oncotarget 8: 104508-104524, 2017.

14. Ye Y, Zhuang J, Wang G, He S, Ni J and Xia W: MicroRNA-139 targets fibronectin 1 to inhibit papillary thyroid carcinoma progression. Oncol Lett 14: 7799-7806, 2017.

15. Romano G and Kwong LN: Diagnostic and therapeutic applications of miRNA-based strategies to cancer immunotherapy. Cancer Metastasis Rev 37: 45-53, 2018.

16. Qadir MI and Faheem A: miRNA: A diagnostic and therapeutic tool for pancreatic cancer. Crit Rev Eukaryot Gene Expr 27: 197-204, 2017

17. Ou C, Sun Z, Li X, Li X, Ren W, Qin Z, Zhang X, Yuan W, Wang J, Yu W, et al: miR-590-5p, a density-sensitive microRNA, inhibits tumorigenesis by targeting YAP1 in colorectal cancer. Cancer Lett 399: 53-63, 2017.

18. Zhou L, Zhao LC, Jiang N, Wang XL, Zhou XN, Luo XL and Ren J: MicroRNA miR-590-5p inhibits breast cancer cell stemness and metastasis by targeting SOX2. Eur Rev Med Pharmacol Sci 21: 87-94, 2017.

19. Livak KJ and Schmittgen TD: Analysis of relative gene expression data using real-time quantitative PCR and the 2(-Delta Delta C(T)) method. Methods 25: 402-408, 2001

20. Zhang Y, Zhang L, Zhang G, Li S, Duan J, Cheng J, Ding G, Zhou C, Zhang J, Luo P, et al: Osteosarcoma metastasis: Prospective role of ezrin. Tumour Biol 35: 5055-5059, 2014.
21. Calin GA, Sevignani C, Dumitru CD, Hyslop T, Noch E, Yendamuri S, Shimizu M, Rattan S, Bullrich F, Negrini M and Croce CM: Human microRNA genes are frequently located at fragile sites and genomic regions involved in cancers. Proc Natl Acad Sci USA 101: 2999-3004, 2004.

22. Li SL, Gao HL, Lv XK, Hei YR, Li PZ, Zhang JX and Lu N: MicroRNA-124 inhibits cell invasion and epithelial-mesenchymal transition by directly repressing Snail2 in gastric cancer. Eur Rev Med Pharmacol Sci 21: 3389-3396, 2017.

23. Song YX, Sun JX, Zhao JH, Yang YC, Shi JX, Wu ZH, Chen XW, Gao P, Miao ZF and Wang ZN: Non-coding RNAs participate in the regulatory network of CLDN4 via ceRNA mediated miRNA evasion. Nat Commun 8: 289, 2017.

24. Cai W, Jiang H, Yu Y, Xu Y, Zuo W, Wang S and Su Z: miR-367 regulation of DOC-2/DAB2 interactive protein promotes proliferation, migration and invasion of osteosarcoma cells. Biomed Pharmacother 95: 120-128, 2017.

25. Cui C and Shi X: miR-187 inhibits tumor growth and invasion by directly targeting MAPK12 in osteosarcoma. Exp Ther Med 14: 1045-1050, 2017.

26. Zhang B, Li Y, Hou D, Shi Q, Yang S and Li Q: MicroRNA-375 inhibits growth and enhances radiosensitivity in oral squamous cell carcinoma by targeting insulin like growth factor 1 receptor. Cell Physiol Biochem 42: 2105-2117, 2017.

27. Kim CW, Oh ET, Kim JM, Park JS, Lee DH, Lee JS, Kim KK and Park HJ: Hypoxia-induced microRNA-590-5p promotes colorectal cancer progression by modulating matrix metalloproteinase activity. Cancer Lett 416: 31-41, 2018.

28. Ma D, Chang LY, Zhao S, Zhao JJ, Xiong YJ, Cao FY, Yuan L, Zhang Q, Wang XY, Geng ML, et al: KLF5 promotes cervical cancer proliferation, migration and invasion in a manner partly dependent on TNFRSF11a expression. Sci Rep 7: 15683, 2017.

29. Yang T, Chen M, Yang X, Zhang X, Zhang Z, Sun Y, Xu B, Hua J, $\mathrm{He} Z$ and Song Z: Down-regulation of KLF5 in cancer-associated fibroblasts inhibit gastric cancer cells progression by CCL5/CCR5 axis. Cancer Biol Ther 18: 806-815, 2017.

30. Zhou W, Song F, Wu Q, Liu R, Wang L, Liu C, Peng Y, Mao S, Feng $\mathrm{J}$ and Chen C: miR-217 inhibits triple-negative breast cancer cell growth, migration, and invasion through targeting KLF5. PLoS One 12: e0176395, 2017.

31. Liu JY, Lu JB and Xu Y: MicroRNA-153 inhibits the proliferation and invasion of human laryngeal squamous cell carcinoma by targeting KLF5. Exp Ther Med 11: 2503-2508, 2016.

32. Ji Q, Xu X, Li L, Goodman SB, Bi W, Xu M, Xu Y, Fan Z, Maloney WJ, Ye Q and Wang Y: miR-216a inhibits osteosarcoma cell proliferation, invasion and metastasis by targeting CDK14. Cell Death Dis 8: e3103, 2017. 